\title{
A força explosiva, velocidade e capacidades motoras específicas em futebolistas juniores amadores: Um estudo correlacional
}

\author{
Explosive strength, velocity and specific motor skills in soccer junior \\ players: A correlational study
}

\author{
M.C. Marques, B. Travassos, R. Almeida
}

\begin{abstract}
RESUMO
Os objectivos deste estudo foram: i) avaliar os níveis de força explosiva dos membros inferiores (manifestação "reflexo - elástico - explosiva"), de velocidade e de capacidades motoras específicas, em futebolistas juniores enquadrados em estruturas desportivas de estatuto não profissional; e, ii) conferir a relação entre as valências avaliadas em futebolistas juniores amadores. Trinta e sete futebolistas juniores, pertencentes a três equipas amadoras foram avaliados através do teste de Sargent, salto horizontal, sprint $30 \mathrm{~m}$, mudança de direcção, drible test e velocidade imprimida à bola no remate. De acordo com os resultados, pode afirmar-se que as significativas correlações verificadas entre distintos movimentos constituem uma "ferramenta" útil para explicar parte do rendimento nesta população. Palavras-chave: força explosiva, velocidade, habilidades motoras específicas, futebolistas juniores amadores
\end{abstract}

ABSTRACT

The main aims of the present study were: a) to evaluate speed and technical skills, and b) to examine the relationship between those measures. Thirty-seven junior soccer players under 19 years of age from three amateur soccer teams were evaluated using vertical and horizontal jump tests, 30m sprint speed test, change of direction test, dribble test and the kicking speed test. The significant correlations found help to understand this population's performance.

Keywords: explosive strength, speed, technical skills, junior amateur soccer players

Submetido: 09.01.2010 | Aceite: 30.06.2010

Mário Cardoso Marques. Departamento de Ciências do Desporto da Universidade da Beira Interior, Covilhã, Portugal; Centro de Investigação em Desporto, Saúde e Desenvolvimento Humano, Vila Real, Portugal. Bruno Travassos e Ruben Almeida. Departamento de Ciências do Desporto da Universidade da Beira Interior, Covilhã, Portugal.

Endereço para correspondência: Mário Cardoso Marques, Departamento de Ciências do Desporto, Universidade da Beira Interior, Convento de Santo António, 6200-001 Covilhã, Portugal.

E-mail: mariomarques@mariomarques.com 
O Futebol é uma modalidade desportiva intermitente que, do ponto de vista fisiológico, é extremamente complexa, com acções específicas que evidenciam uma tipologia de esforço de grande diversidade e que metabolicamente apela a diferentes sistemas energéticos (Rebelo \& Oliveira, 2006). A necessidade de desencadear esforços curtos e intensos parece ser um imperativo lógico para que o jogador de futebol realize com sucesso um conjunto de acções críticas como a corrida rápida (sprint) executada com mudanças de sentido ou direcção, variações de velocidade, travagens ou arranques bruscos, pontapés de baliza, cantos, livres, saltos, "tackles", remates e outros gestos que façam um apelo específico à capacidade de produzir força (Bangsbo, 1997).

Embora alguns autores considerem a força como uma variável determinante para elevar o rendimento específico do futebolista (e.g., Marques, 2004), parece-nos ser necessário mais estudos sobre esta temática. Por outro lado, apesar de vários estudos (e.g., de Proft, Cabri, Duffour, \& Clarys, 1988) tivessem demonstrado que a aplicação de programas de desenvolvimento da força explosiva têm uma implicação directa na melhoria de aç̧ões próprias do futebol (remate, sprint...), parece estar longe o consenso acerca do grau de associação, já que existem vários estudos com resultados algo diferenciados, recorrendo aos mesmos princípios metodológicos, tal como referem Seabra, Maia e Garganta (2001).

A manifestação da força explosiva é fundamental durante as múltiplas acelerações, mudanças "bruscas" de direcção com e sem bola, e crucial para uma melhor impulsão vertical para cabecear com maior eficácia. Esta capacidade motora é igualmente decisiva para o desenvolvimento de um conjunto de execuções técnicas da modalidade e para uma eficiente inclusão de acções tácticas (Rebelo \& Oliveira, 2006). De facto, a força explosiva está fortemente associada a uma panóplia de acções de carácter veloz. Porém, sobretudo nas acções de elevada intensidade e de curta duração, perduram questões controversas quando se refere a relação entre a força explosiva e a velocidade, e destas com a performance em habilidades como o remate ou o drible.

Segundo Sousa, Garganta, e Garganta (2003), vários autores têm-se debruçado sobre a relação entre a força explosiva dos membros inferiores e a velocidade máxima imprimida à bola durante o remate, ainda que este tema tenha sido medianamente estudado e, como tal, ainda controverso. Sobre esta temática, alguns autores encontraram uma associação positiva, mas não significativa, entre a força e estes gestos técnicos, enquanto outros estudos reportaram resultados antagónicos (Sousa et al., 2003). Martínez, Salgado, Lago, e Peñas (2004) examinaram a relação entre a força explosiva dos membros inferiores e a capacidade de aceleração linear com as mudanças de direcção. Os autores puderam observar uma correlação positiva e significativa, sendo esta maior quando as distancias eram menores e em percursos com mudanças sucessivas de direcção. Em contrapartida, Gonzalez e Aguiar (2001) não constataram qualquer associação entre a corrida linear de curta duração (30 metros) e a força explosiva dos membros inferiores.

Para além das divergências salientadas, subsistem ainda na literatura lacunas relevantes sobretudo no que diz respeito à avaliação destas habilidades motoras em jogadores de futebol dos escalões de Juniores. Na última década, têm sido publicados estudos no âmbito da caracterização cineantropométrica e condicional dos futebolistas, mas quase todos se referem ao modelo de rendimento em futebolistas profissionais (Martínez et al., 2004). Neste sentido, parecenos relevante a avaliação das capacidades condicionais em jogadores não só do escalão Sénior, mas procurando a caracterização dos jogadores de Futebol em escalões etários mais jovens, especificamente no escalão Júnior. Atendendo a este enquadramento, objectiva-se no presente estudo: i) Avaliar os níveis de força explosiva dos membros inferiores, os índices de velocidade de curta duração, e 
mensurar acções motoras específicas, em futebolistas Juniores enquadrados em estruturas desportivas de estatuto não profissional; e, ii) Identificar o grau de associação entre os níveis de força explosiva dos membros inferiores com sprint, mudanças de direcção, drible e o remate.

\section{MÉTODO}

\section{Amostra}

A amostra foi constituída por trinta e sete praticantes de Futebol $(n=37)$, do sexo masculino, com idades compreendidas entre os 16 e os 19 anos de idade, saudáveis e abrangendo todas as posições específicas. Os indivíduos pertenciam a 3 equipas do escalão Júnior (sub-19). Na Tabela 1 pode ver-se com detalhe a caracterização da amostra.

Tabela 1

Características dos indivíduos $(n=37)$ pertencentes à amostra em estudo

\begin{tabular}{lrrrr}
\hline \multicolumn{1}{c}{ Variáveis } & Min. & Máx. & \multicolumn{1}{c}{ M } & DP \\
\hline Idade $($ anos $)$ & 16.0 & 19.0 & 17.41 & .96 \\
Altura $(\mathrm{cm})$ & 168.0 & 183.0 & 173.81 & 4.27 \\
Peso $(\mathrm{kg})$ & 60.1 & 78.0 & 67.19 & 4.65 \\
IMC $\left(\mathrm{m} / \mathrm{kg}^{2}\right)$ & 20.1 & 24.0 & 22.23 & 1.11 \\
\hline Nota: IMC = índice de massa corporal & &
\end{tabular}

\section{Instrumentos e Procedimentos}

Para avaliar a força explosiva das extremidades inferiores, a velocidade e a capacidade de realizar acções motoras específicas do Futebol, foram seleccionados os seguintes testes: Sargent e salto horizontal, sprint de $30 \mathrm{~m}$, deslocamento com mudança de direcção, drible test e velocidade de remate.

Todos os protocolos de avaliação foram aplicados durante a fase final do período competitivo compreendido entre Março e Abril. Antes da execução dos diferentes testes, todos os jogadores realizaram o habitual aquecimento, orientado pelo respectivo treinador. Todos os sujeitos foram previamente familiarizados com os procedimentos de cada um dos testes. A ordem de realização dos testes correspondeu à sequência de apresentação dos respectivos procedimentos que são descritos nos próximos parágrafos.

\section{Indicadores de força explosiva}

Para avaliar a força explosiva foram seleccionados e aplicados os seguintes protocolos de avaliação: teste de Sargent (SAR) e o salto horizontal (SH).

Sargent (SAR). Na execução deste teste foram adoptados os seguintes procedimentos: a) o executante de pé de frente para uma parede; b) com os braços ao lado do corpo e as plantas dos pés totalmente apoiadas no solo; c) e inicialmente com a ponta dos pés a tocarem a parede. O executante estendia ambos os braços acima da sua cabeça marcando a parede com a ponta dos dedos de modo a registar a altura inicial. Para tal, a ponta dos dedos de uma das mãos foi impregnada com pó colorido e humedecida em água. Em seguida, mantendo os braços para cima, afastava-se cerca de $30 \mathrm{~cm}$ da parede colocando-se lateralmente à mesma. Tomava impulso por meio de uma semi-flexão dos joelhos (até ao momento em que realiza um ângulo de $90^{\circ}$ ao nível das articulações dos joelhos). Para potenciar o salto podia utilizar os braços para alcançar a altura máxima. No momento em que o executante alcançava a altura máxima deveria tocar a parede com os dedos de forma a registar o incremento na altura face à posição inicial (afixou-se na parede uma escala métrica). A altura vertical do salto foi determinada pelo cálculo da diferença entre a altura da marca no salto e a marca da altura inicial. Cada indivíduo executou 2 tentativas, com intervalo de 2 minutos, tendo-se registado ambas as tentativas e calculado a sua média.

Salto horizontal (SH). Durante a realização deste teste seguimos os seguintes passos: a) cada indivíduo encontrava-se de pé com os apoios colocados paralelamente a uma marca (zero) de uma escala métrica fixada no chão; b) em seguida cada indivíduo realizava o movimento de semi-flexão dos joelhos, podendo auxiliar este gesto com os braços para saltar o mais distante possível, procurando, na 
fase final, manter o equilíbrio e os apoios sempre paralelos; c) mediu-se a distância da marca zero até ao ponto mais próximo alcançado pelo calcanhar do apoio durante a queda. Todos os participantes realizaram 2 tentativas, com 2 minutos de intervalo, registando-se a sua média.

\section{Indicadores de velocidade}

De forma a avaliar a velocidade de deslocamento foram seleccionados e aplicados o teste de corrida linear, Sprint $30 \mathrm{~m}$ (S30), e um teste de deslocamento com mudança de direcção (MD). Para a realização destes testes, os jogadores utilizaram o seu calçado de competição, pois estes foram realizados em relva sintética. Utilizaram-se dois cronómetros digitais manuais, sendo o valor final de cada tentativa igual à média dos dois valores registados em cada tentativa.

Sprint $30 \mathrm{~m}$ (S30). A corrida iniciava-se com o indivíduo assumindo uma posição vertical em situação estática e com um dos apoios mais adiantado. Todos os segmentos corporais encontravam-se atrás da linha inicial do percurso $(3 \mathrm{Om})$. O sinal de partida foi dado de forma sonora. Cada indivíduo executou 2 tentativas, com um intervalo de 5 minutos, tendo-se registado ambas as tentativas e calculado a sua média.

Deslocamento com mudança de direcção (MD). A fase inicial deste teste foi idêntica ao S30. Após o sinal sonoro, cada participante teve de percorrer à máxima velocidade possível num espaço total de $15 \mathrm{~m}$, com mudanças de direcção (com um ângulo de $90^{\circ}$ ) a cada $5 \mathrm{~m}$, para a direita e para a esquerda, respectivamente. Cada indivíduo executou 2 tentativas, com um intervalo de 5 minutos, registando-se ambas as tentativas e calculada a sua média.

\section{Indicadores das habilidades motoras específicas}

Neste âmbito, os testes pretenderam descrever o nível de aptidão nas habilidades de dribling, através do drible test (DT) e velocidade de remate (VR). Para a realização destes testes foi utilizada uma bola oficial de Futebol Adidas Roteiro $^{\mathrm{TM}}$, cuja circunferência e peso estavam compreendidas entre os 68 e $70 \mathrm{~cm}$, e as 410 e $450 \mathrm{~g}$, respectivamente, conforme o estipulado na Lei 2 do quadro das leis de jogo de Futebol de 11, publicadas pela FIFA. Os indivíduos calçaram o seu calçado de competição e o tipo de terreno foi constituído pelo mesmo piso já mencionado. Para a realização destes testes os jogadores utilizaram uma vez mais o calçado de competição pela razão supracitada.

Drible test (DT). Neste teste, os jogadores tiveram de driblar a bola em redor de quatro cones, distantes entre si de 3.5 metros, descrevendo uma trajectória em forma de oito, o mais rápido possível. Foi medido o tempo gasto na execução da tarefa. O sinal de partida foi constituído por sinal sonoro. A medição foi a mesma que se utilizou para o S30. Cada indivíduo executou 2 tentativas, com um intervalo de 5 minutos, tendo-se registado ambas as tentativas e calculado a sua média.

Velocidade de remate (VR). O remate foi efectuado com a bola parada, encontrando-se esta a uma distância de 11 metros da linha de baliza e na perpendicular do ponto que separava esta linha em duas metades iguais (marca da grande penalidade). Cada executante foi encorajado a realizar o remate à máxima velocidade (objectivo) com eficácia (a bola entrar na baliza era condição para validação da situação). A velocidade de saída da bola foi medida através de um radar (Stalker Profissional Sports Radar), calibrado da seguinte forma: Setup menu: Ball Lo 16; Range: Low. O radar foi colocado atrás da posição inicial da bola, no mesmo plano de deslocamento da bola em relação à baliza $\left(0^{\circ}\right)$, diminuindo o erro associado (erro de $.013 \mathrm{~m} / \mathrm{s}$ ). Os jogadores dispuseram de 2 tentativas, com intervalo de repouso de 2 minutos entre tentativas, tendose registado ambas as tentativas e calculado a sua média.

\section{Fiabilidade dos testes}

Os resultados deste estudo podem ser observados na Tabela 2. Destaca-se a elevada 
fiabilidade dos testes para as variáveis de interesse aqui analisadas.

Tabela 2

Resultados sobre o estudo de fiabilidade dos testes

\begin{tabular}{lcc}
\hline \multicolumn{1}{c}{ Testes } & CCI & Intervalo \\
\hline Sargent & .98 & $.98-.99$ \\
Salto horizontal & .98 & $.94-.98$ \\
Sprint 30 m & .80 & $.64-.89$ \\
Deslocamento com & .92 & $.85-.96$ \\
mudança de direcção & & $.70-.91$ \\
Drible test & .83 & $.94-.97$ \\
Velocidade de remate & .97 & .94 \\
\hline
\end{tabular}

Nota: $\mathrm{CCI}=$ coeficiente de correlação intra-classe

\section{Análise estatística}

Para o tratamento dos dados foram utilizados procedimentos estatísticos descritivos, como a média aritmética e o desvio-padrão, e preditivos, como o coeficiente de correlação de Pearson. O programa estatístico utilizado foi o SPSS 15.0, tendo-se aceite um nível de confiança de $95 \%(p<.05)$.

\section{RESULTADOS}

A Tabela 3 apresenta os valores médios e respectivos desvios-padrão dos indicadores da força explosiva (SAR e SH), dos indicadores de velocidade (capacidade de arranque) (S30 e MD) e ainda dos indicadores de capacidade de realizar acções motoras específicas (DT e VR).

Tabela 3

Resultados sobre todos os testes realizados

\begin{tabular}{lrr}
\hline \multicolumn{1}{c}{ Testes } & \multicolumn{1}{c}{ M } & \multicolumn{1}{c}{ DP } \\
\hline Sargent $(\mathrm{cm})$ & 43.60 & 3.78 \\
Salto horizontal $(\mathrm{m})$ & 2.05 & .12 \\
Sprint 30 m $(s)$ & 4.50 & .13 \\
Deslocamento com & 4.01 & .15 \\
mudança de direcção $(s)$ & & \\
Drible test $(\mathrm{s})$ & 8.87 & .52 \\
Velocidade de remate $(\mathrm{m} / \mathrm{s})$ & 26.30 & 1.63 \\
\hline
\end{tabular}

Os coeficientes de correlação de Pearson são apresentados na Tabela 4. O teste de SAR evidenciou correlações significativas com o SH, com o teste de MD e com o S30 $(r=.823$, $p<.01 ; r=-.423, p<.01 ; \mathrm{e}, r=-.408, p<.05$, respectivamente). Por sua vez, o teste do $\mathrm{SH}$ demonstrou uma correlação significativa com o teste de mudança de direcção $(r=-.423$, $p<.01)$ e o sprint com a mudança de direcção $(r=.347, p<.05)$.

Tabela 4

Resultados sobre coeficientes de correlação de Pearson

\begin{tabular}{|c|c|c|c|c|c|}
\hline & SAR & $\mathrm{SH}$ & S30 & MD & DT \\
\hline Sargent (SAR) & - & & & & \\
\hline Salto horizontal (SH) & $.823^{* *}$ & - & & & \\
\hline Sprint $30 \mathrm{~m}(\mathrm{~S} 30)$ & $-.408^{*}$ & -.299 & - & & \\
\hline Desl. com mudança de direcção (MD) & $-.423^{* *}$ & $-.474^{* *}$ & $.347^{*}$ & - & \\
\hline Drible test (DT) & .149 & .009 & -.150 & -.019 & - \\
\hline Velocidade de remate (VR) & .136 & .016 & -.012 & -.031 & .073 \\
\hline
\end{tabular}

\section{DISCUSSÃO}

Este estudo teve como principais objectivos examinar os níveis de força explosiva em jovens jogadores de Futebol e verificar o grau de associação entre os mesmos. Neste capítulo, a literatura é escassa especialmente no nosso País. Todavia, destacamos as correlações significativas, ainda que preferencialmente moderadas, entre o salto horizontal e a impulsão vertical, bem como entre esta última com o sprint. De referir ainda a inexistência de qualquer relação entre a impulsão vertical e o remate.

Relativamente ao teste de Sargent, os resultados encontrados no presente estudo foram semelhantes aos valores percebidos noutros trabalhos com populações semelhantes (Martínez et al., 2004; Resende, Fornaziero, Cunha, \& Osiecki, 2007). Em relação ao salto horizontal, os valores registados foram 
inferiores aos observados por Resende et al. (2007), Seabra et al. (2001) e Toledo (2005), mas similares aos encontrados por Ré, Bojikian, Teixeira, e Böhme (2005), todavia em desportistas mais jovens (15.8 \pm .6 anos). No que diz respeito ao teste $\mathrm{S} 30$, os resultados verificados neste estudo $(4.50 \quad \pm \quad .13 \mathrm{~s})$ apresentam níveis inferiores aos encontrados noutras investigações (Martínez et al., 2004; Resende et al., 2007). No entanto, Toledo (2005) e Ré et al. (2005) registaram valores mais baixos comparativamente ao nosso estudo, mas em jogadores ligeiramente mais jovens. Em suma, parece-nos que não existe uma uniformidade nos valores de força explosiva, quer em testes específicos, quer não específicos, em jovens jogadores de Futebol.

Para os testes MD e DT, apesar da literatura os considerar válidos para o objectivo e âmbito deste estudo, os protocolos publicados variam em termos de distâncias utilizadas. Por outro lado, nos estudos descritos, as amostras não são coincidentes com a considerada neste estudo. Para a variável velocidade de remate (VR), foram apurados, no presente estudo, os seguintes valores médios: $26.30 \pm 1.63 \mathrm{~m} / \mathrm{s}$ (Tabela 3). Estes valores são inferiores aos encontrados por diferentes estudos realizados em populações idênticas (Sousa et al., 2003), bem como em jogadores Seniores (Cometti, Maffiuletti, Pousson, Chatard, \& Maffulli, 2001). Segundo Luhtanen (1994), as velocidades de remate estão compreendidas entre os 17 e $28 \mathrm{~m} / \mathrm{s}$, onde jogadores Seniores de elite podem atingir valores entre os 32 e os $35 \mathrm{~m} / \mathrm{s}$. Assim, verifica-se que as velocidades de remate observadas neste estudo são similares a outros previamente publicados.

Podemos sugerir que a discrepância dos valores percebidos no nosso estudo pode ser parcialmente explicada através das diferenças entre as metodologias de avaliação adoptadas em cada um dos estudos, nos tipos de população utilizadas, e nos diferentes níveis de treino dos participantes.

Ainda que os estudos em Futebol sejam escassos quando se trata de abordar associações entre variáveis de performance com características explosivas, o presente trabalho revelou importantes relações a considerar. Destacamos a relação entre o $\mathrm{SH}$ e o SAR, entre o sprint e o mesmo salto vertical, assim como com as mudanças rápidas de direcção. Em todos estes movimentos está presente $\mathrm{o}$ ciclo do alongamento e encurtamento, o que pode explicar o elevado grau de dependência entre estas variáveis (Marques, 2004). Estes dados são determinantes aquando da planificação de programas de condição física em jovens futebolistas. Todavia, estas relações apenas nos indicam o grau de associação entre variáveis de rendimento e nunca poderão ser entendidas como a única fonte de explicação para as alterações de performance. Tal como foi observado (ver Tabela 4), o teste de SAR mostrou uma associação significativa mas moderada com o sprint (S30) $(r=-.408, p<.05)$ e uma forte correlação com o salto horizontal (SH) $(r=.823, p<.01)$, bem como uma relação significativa, ainda que moderada, com o teste de mudança de direcção (MD) $(r=-.423$, $p<.01)$. O SH evidenciou, ainda, uma associação com o MD $(r=-.474, p<.01)$. Gonzalez e Aguiar (2001) verificaram uma associação positiva $(r=.377)$ entre o S30 e o MD em jogadores de futebol com idades ligeiramente inferiores (15.5 \pm .5 anos) às do nosso estudo. Por outro lado, Rebelo e Oliveira (2006) observaram uma relação superior entre os testes de corrida linear e de corrida com fortes mudanças de direcção, em jogadores profissionais. No que se refere à associação entre o SH e o teste S30, Toledo (2005) verificou um coeficiente de correlação semelhante ao encontrado neste estudo $(r=-.299, p<.01)$.

De salientar o facto de o drible test não ter demonstrado nenhuma correlação significativa com o desempenho nos restantes testes. Isto parece sugerir que o desempenho na execução da condução da bola num percurso não linear, não está apenas dependente das capacidades condicionais, mas sobretudo de aspectos 
relacionados com a capacidade perceptivomotora dos indivíduos (Soares, 2005).

No presente estudo não se detectou qualquer correlação entre o salto vertical e a velocidade de remate, corroborando o estudo de Sousa et al. (2003). Assim, apesar de o remate ser um movimento de natureza explosiva, o nível de controlo coordenativo parece assumir-se como o factor preponderante na performance do mesmo, relativamente ao parâmetro velocidade imprimida à bola. Sousa et al. (2003) acrescentam que a relação entre salto vertical e a velocidade do remate tende a aumentar com o número de anos de prática desportiva. Esse facto poderá ser explicado por um efeito de aprendizagem do controlo do movimento, o que induz um melhor aproveitamento dos recursos biomecânicos.

O período pubertário masculino é caracterizado por um aumento percentual da massa muscular dos indivíduos, acompanhado de um desenvolvimento superior da capacidade anaeróbica (Seabra et al., 2001). Segundo Marques (2004), nesta fase dá-se o aperfeiçoamento final do padrão físico do indivíduo ao nível da coordenação motora, da flexibilidade, da velocidade e da força muscular. Deste modo, podemos afirmar que na idade Júnior, os indivíduos possuem um biótipo mais definido, encontrando-se numa fase de especialização da modalidade. Contudo, neste escalão, enquadram-se frequentemente jogadores do escalão inferior (Juvenis), os quais se encontram em estados de maturação diferentes face à individualidade biológica. Assim, é necessário uma correcta avaliação da capacidade de cada indivíduo para que possamos definir e quantificar o sinal de entrada e desenhar um sistema de controlo que permita aferir a relação entre a carga real e a carga proposta, atendendo ao défice de adaptação de cada desportista.

Através deste estudo ficou patente a necessidade de implementar um maior número de testes para avaliar a capacidade motora específica, sobretudo aqueles que sejam capazes de mensurar diferentes indicadores de performance no Futebol e que contribuam para uma melhor caracterização da modalidade. Por exemplo, a velocidade máxima de remate observada neste estudo é inferior a outras investigações semelhantes, mas os valores aqui percebidos encontram-se dentro da amplitude de velocidades aceitáveis para esta faixa etária (Luhtanen, 1994).

Fica ainda em aberto a discussão da relação entre a capacidade de manifestação de força explosiva dos membros inferiores $\mathrm{e} a$ velocidade de remate. Sugerimos que em estudos posteriores se procure a aplicação de protocolos de avaliação de salto que empreguem instrumentos mais sofisticados, como por exemplo, o uso de plataformas de força que ofereçam mais informação nos parâmetros derivados da curva força-tempo resultante.

Para finalizar, realce-se ainda a necessidade de uma melhor compreensão da relação entre força e velocidade e os aspectos relacionados com as capacidades perceptivo-motoras. Como verificámos, o drible test não obteve qualquer correlação com os restantes parâmetros avaliados. Sendo esta capacidade um factor determinante nesta modalidade, torna-se necessário a validação de testes que associem os factores condicionais observados com os factores perceptivo-motores associados ao jogo.

\section{REFERÊNCIAS}

Bangsbo, J. (1997). Entrenamiento de la condición fisica en el fútbol. Barcelona: Editorial Paidotribo.

Cometti, G., Maffiuletti, N. A., Pousson, M., Chatard, J. C., \& Maffulli, N. (2001). Isokinetic strength and anaerobic power of elite, subelite and amateur French soccer players. International Journal of Sports Medicine, 22 (1), 45-51.

de Proft, E., Cabri, J., Duffour, W., \& Clarys, J. (1988). Strength training and kick performance in soccer players. In T. Reilly, A. Lees, K. Davids, \& W. J. Murphy (Eds.), Science and football (pp. 108-113). London: E. \& F. N. SPON.

Gonzalez, M., \& Aguiar, S. (2001). Estudio comparativo entre a carrera lineal e con cambios de 
dirección e a su relación con valores do teste de Bosco. Vigo: Universidad de Ciencias de Educación de Pontevedra e Universidad de Vigo.

Luhtanen, P. (1994). Jumping in soccer. Science $\mathcal{E}$ Football, 8, 11-13.

Marques, M. C. (2004). O trabalho de força no alto rendimento desportivo: Da teoria à prática. Lisboa: Livros Horizonte.

Martínez, L. C., Salgado, J. J. C., Lago, E. D., \& Peñas, C. L. (2004, Março). Relación entre parámetros antropométricos y manifestaciones de fuerza $y$ velocidad en futbolistas en edades de formación. Comunicação apresentada no III Congreso de la Asociación Española de Ciencias del Deporte, Valência, Espanha.

Ré, A. H. N., Bojikian, L. P., Teixeira, C. P., \& Böhme, M. T. S. (2005). Relações entre crescimento, desempenho motor, maturação biológica e idade cronológica em jovens do sexo masculino. Revista Brasileira de Educação Física e Esporte, 19(2), 153-162.

Rebelo, A. N., \& Oliveira, J. (2006). Relação entre a velocidade, a agilidade e a potência muscular de futebolistas profissionais. Revista Portuguesa de Ciências do Desporto, 6(3), 342-348.
Resende, F., Fornaziero, M., Cunha, C., \& Osiecki, R. (2007, Outubro). Aspectos neuromusculares, antropométricos e funcionais em atletas de futebol da categoria juvenil. Comunicação apresentada no XXX Simpósio Internacional de Ciências do Desporto, São Paulo, Brasil.

Seabra, A., Maia, J. A., \& Garganta, R. (2001). Crescimento, maturação, aptidão física, força explosiva e habilidades motoras específicas: Estudo em jovens futebolistas e não futebolistas do sexo masculino dos 12 aos 16 anos de idade. Revista Portuguesa de Ciências do Desporto, 1 (2), 22-35.

Soares, J. (2005). O treino do futebolista (vol. 1). Porto: Porto Editora.

Sousa, P., Garganta, J., \& Garganta, R. (2003). Estatuto posicional, força explosiva dos membros inferiores e velocidade imprimida à bola no remate: Um estudo com jovens praticantes do escalão sub-17. Revista Portuguesa de Ciências do Desporto, 3 (3), 27-35.

Toledo, N. D. (2005, Dezembro). Força rápida, força explosiva e velocidade de deslocamento em futebolistas profissionais: Um estudo diagnóstico. Comunicação apresentada no I Congresso de Ciências do Desporto, Campinas, São Paulo. 